\title{
The Cooling of a Liquid Absorber using a Small Cooler*
}

\author{
D. E. Baynham ${ }^{1}$, T. W. Bradshaw ${ }^{1}$, M. A. Green ${ }^{2}$, \\ S. Ishimoto ${ }^{3}$, and N. Liggins ${ }^{2}$
}

1. CCLRC Rutherford Appleton Laboratory, Chilton-Didcot, OX11-0QX, UK

2. Lawrence Berkeley National Laboratory, Berkeley CA 94703, USA

3. KEK Laboratory, 1-1 Oho, Tsukuba, 305-0801, Japan

\section{August 2005}

Presented at the 2005 Cryogenic Engineering Conference

Keystone Colorado, USA

29 August through 2 September 2005

Published in Advances in Cryogenic Engineering 51

\footnotetext{
* This work was supported by the Oxford University Physics Department and the Particle Physics and Astronomy Research Council of the United Kingdom. A portion of this work was done with the support of the Office of High Energy Physics, United States Department of Energy under DOE contract DE-AC02-05CH11231.
} 


\title{
THE COOLING OF A LIQUID ABSORBER USING A SMALL COOLER
}

\author{
D. E. Baynham ${ }^{1}$, T. Bradshaw ${ }^{1}$, M. A. Green ${ }^{2}$, S. Ishimoto ${ }^{3}$, and N. Liggins ${ }^{2}$ \\ ${ }^{1}$ CCLRC Rutherford Appleton Laboratory ASTeC, \\ Chilton-Didcot, OX11-0QX, UK \\ ${ }^{2}$ Lawrence Berkeley National Laboratory \\ Berkeley CA 94720, USA \\ ${ }^{3}$ KEK Laboratory, 1-1 Oho, \\ Tsukuba, 305-0801, Japan
}

\begin{abstract}
This report discusses the use of small cryogenic coolers for cooling the Muon Ionization Cooling Experiment (MICE) liquid cryogen absorbers. Since the absorber must be able contain liquid helium as well liquid hydrogen, the characteristics of the available $4.2 \mathrm{~K}$ coolers are used here. The issues associated with connecting two-stage coolers to liquid absorbers are discussed. The projected heat flows into an absorber and the cool-down of the absorbers using the cooler are presented. The warm-up of the absorber is discussed. Special hydrogen safety issues that may result from the use of a cooler on the absorbers are also discussed.
\end{abstract}

KEYWORDS: Small 4 K Coolers, $\mathrm{LH}_{2}$, LHe, and Hydrogen Safety

PACS: 07.20Mc, 65.20+w, 06.60Wa

\section{INTRODUCTION}

The original MICE proposal [1] called for cooling the MICE liquid hydrogen absorbers from a central refrigerator [2], which would also cool the magnets and light detectors. In this approach, cooling would be delivered to the hydrogen absorbers as helium gas at $14 \mathrm{~K}$ to a heat exchanger on the absorber tank. A problem with the MICE central refrigeration system was that most of the refrigeration is needed to cool transfer lines. The cost of a central refrigeration system, which includes the cold box, the compressors, a control system and the site infrastructure needed for power and cooling the helium compressor, was considered to be excessive. In addition, refrigeration would be needed to test the superconducting solenoids and the absorbers before shipment to the MICE site.

It was suggested that about twenty two-stage $4-\mathrm{K}$ coolers could replace the large refrigeration plant to cool the magnets [3], the absorbers [4], and the detector VLPCs. The use of small coolers is a more cost effective solution. The small cooler option permits one to mount the coolers on the MICE modules at the manufacturers site. Cool-down and testing of the magnets can occur at the magnet supplier's plant before the magnet modules are shipped to the Rutherford Appleton Laboratory (RAL) for final assembly into the MICE channel. 
Cool-down and testing of the liquid absorbers would be done at RAL before they are installed in the MICE channel. Another benefit of the use of small coolers is that large capital expenditure for MICE refrigeration does not have to be made at the beginning of the project. The refrigeration costs can be staged over the construction phase of the project.

Small coolers have the advantage that they deliver refrigeration at 4 to $20 \mathrm{~K}$ on the second stage while delivering refrigeration at 40 to $60 \mathrm{~K}$ simultaneously at the first stage. First stage cooling can be used to pre-cool the hydrogen gas that will be liquefied in the absorber as it is being filled. The first stage of the cooler also intercepts the heat coming down the various pipes and instrumentation leads that come into the absorber.

The first key element to being able to cool the liquid absorbers with small coolers is reducing the heat leak into the absorber sufficiently that it can be operated with liquid helium. This report will show how the heat leak into the absorbers can be reduced to $<1 \mathrm{~W}$ at $4 \mathrm{~K}$. The connection of the small cooler to the absorber is the second key element in the successful use of small cooler to cool the liquid absorbers. The reduction of the $\square \mathrm{T}$ between the absorber and the cooler second stage cold head is particularly important when the absorber is filled with liquid helium. The third key element is liquid hydrogen (or helium) safety. The effects on safety of using a small cooler to cool a liquid absorber will be discussed.

\section{THE COOLER REQUIREMENTS FOR MICE ABSORBERS}

Since the MICE liquid absorbers are designed to operate with either liquid helium or liquid hydrogen, the absorbers must be cooled using $4 \mathrm{~K}$ coolers [5]. Commercial coolers that operate at $4 \mathrm{~K}$ are currently only available from Sumitomo Industries and Cryomech. There are a several companies that produce small two stage coolers that will operate at $15 \mathrm{~K}$ but these machines are smaller than desired. They are the RDK-415D (a two-stage 4-K GM cooler) from Sumitomo [6] and the PT-410 (a two-stage 4-K pulse tube cooler) from Cryomech [7].

The pulse-tube cycle used by Cryomech is thermodynamically similar to the cycle of a Gifford McMahon (GM) cooler. The pulse tubes used in the Cryomech machine are lowfrequency (about $3 \mathrm{~Hz}$ ) pulse tubes. In order for the pulse tubes to operate at low frequencies, there are slide valves in the cold-head assembly. Cryomech has apparently solved the leakage problem that some manufacturers have had with these valves. As a result there appear to be very few breakdowns of these machines, due to the slide valves.

The only moving part in the pulse tube machine cold-head assemblies are the warm slide valves whereas the GM machines have moving pistons in the cold heads. As a result, the vibration acceleration delivered by one GM machine (standing alone) is of the order of $0.1 \mathrm{~g}$. The pulse tube machines have vibration accelerations that are two orders of magnitude lower than the GM machines. The MICE absorbers are not affected by vibration so either type of cooler can be used..

A key question is whether the pulse tube machines are significantly more reliable than the GM machines. There is not an absolutely clear answer to this question. The RDK-415D machine has a shorter cold head maintenance interval than does the PT-410 machine. On the other hand, at least one laboratory in the US has run an RDK-415D continuously since 1999. (The problem with exceeding the recommended maintenance interval may be the voiding the warranty on the machine.) Based on what is known today, either machine appear to be more than reliable enough to cool the MICE liquid absorbers.

It shall become clear later in this report that the MICE absorbers can be cooled using either pulse-tube coolers or GM coolers. The decision as to which type of cooler to use may hinge on issues such as maintenance, the temperature margin in the absorber or magnet at liquid helium temperatures, or the availability of cooling water in the MICE experimental hall.

FIGURE 1 is a typical performance curve (for both stages) for a 1 to $1.2 \mathrm{~W}$ (at $4.2 \mathrm{~K}$ ) pulse tube cooler that operates over a temperature range from $2.5 \mathrm{~K}$ to $20 \mathrm{~K}$ at the second stage and $25 \mathrm{~K}$ to $80 \mathrm{~K}$ at the first stage. The input power to the compressor is at $50 \mathrm{~Hz}$. A typical 1.5-W GM cooler performance curve is similar to the one shown in FIG. 1, except that the heat load at the first stage has a smaller effect on the refrigeration that can be delivered by the second-stage of the cooler. For the MICE absorber, where there is a relatively low heat load into the cooler first-stage, the performance of the two types of coolers would be similar. 


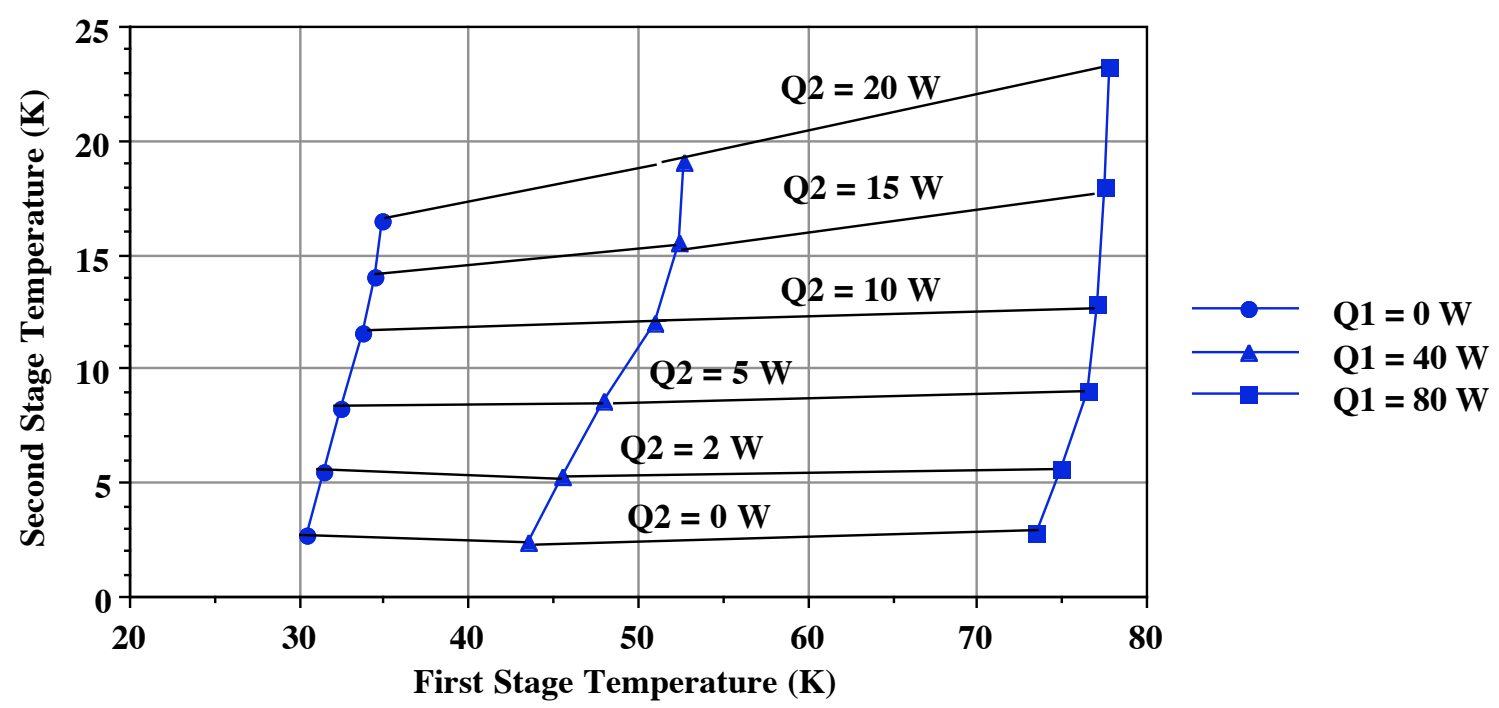

FIGURE 1. A typical $4 \mathrm{~K}$ pulse tube cooler performance curve, which shows the first and second stage temperatures as a function of various heat loads onto the first stage Q1 and second stage Q2 of the cooler. The performance curves for the GM cooler should be flatter for high first-stage heat loads. This curve is for reference purposes only and does not apply to a particular cooler. One must contact the cooler manufacturer for the desired cooler performance curves over a broad range heat loads on both stages.

\section{THE HEAT LOADS FOR THE MICE LIQUID ABSORBER}

The heat loads into the absorber come from three sources; 1) radiation heating into the absorber body, the thin windows and the pipes and strap coming into the absorber, 2) conduction heat loads through the absorber supports in both the longitudinal and radial directions, and 3) conduction through the pipes and instrumentation wires. FIGURE 2 shows a three-dimensional representation of the MICE liquid absorber with its condenser and surge volume and the $4 \mathrm{~K}$ cooler used to keep the absorber cold. The table within FIG. 2 shows the absorber dimensions needed to calculate the heat leak into the MICE absorber by radiation and conduction.

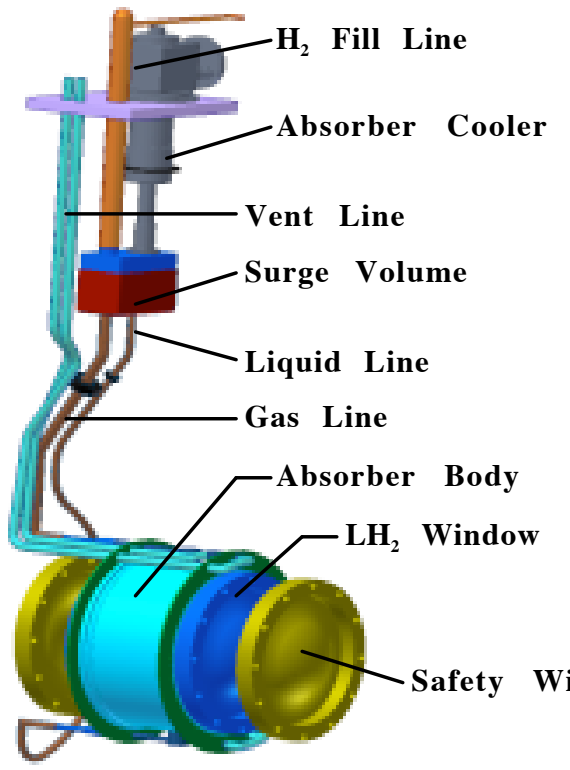

\section{Parameter}

\begin{tabular}{lc}
\hline \hline Absorber body outside diameter $(\mathrm{mm})$ & $\mathbf{3 8 0}$ \\
Absorber thin window diameter $(\mathrm{mm})$ & $\mathbf{3 0 0}$ \\
Absorber body length $(\mathrm{mm})$ & $\mathbf{2 8 0}$ \\
Absorber body surface area $\left(\mathrm{m}^{2}\right)$ & $\mathbf{0 . 5 1}$ \\
Thin window surface area $\left(\mathrm{m}^{2}\right)$ & $\mathbf{0 . 1 4}$ \\
Absorber feed tube length $(\mathrm{m})$ & $\sim \mathbf{2 . 2}$ \\
Surge tank outside diameter $(\mathrm{mm})$ & $\mathbf{1 5 0}$ \\
Surge tank outside length $(\mathrm{mm})$ & $\mathbf{3 0 0}$ \\
Pipe, Cu strap, and tank area $\left(\mathrm{m}^{2}\right)$ & $\sim \mathbf{0 . 3 2}$ \\
\hline
\end{tabular}

FIGURE 2. A three-dimensional representation of the MICE liquid absorber, the condenser, and the absorber $4 \mathrm{~K}$ cooler. The table that is part of this figure, presents the basic parameters of the MICE liquid absorber, and its system of pipes. These parameters can be used to calculate the absorber heat load. 
TABLE 1. A summary of the heat loads into the MICE liquid absorber with 10 layers of MLI on the absorber body, the pipes, strap, and liquid buffer volume; and with 4 layers of MLI across the thin windows.

\begin{tabular}{lcc}
\multicolumn{1}{c}{ Parameter } & Shield & Absorber \\
\hline \hline 16 layers of MLI on the body \& pipes (W) & -- & $\sim 0.45$ \\
4 Layers of MLI on the thin windows (W) & -- & $\sim 0.35$ \\
20 Layers of MLI in neck region (W) & $\sim 1.0$ & --- \\
Cold mass support heat leak (W) & -- & $\sim 0.20$ \\
Instrumentation lead heat load (W) & 0.15 & $\sim 0.01$ \\
Piping and duct heat load (W) & 2.36 & $\sim 0.1$ \\
Total heat load into the absorber (W) & $\sim 3.5$ & $\sim 1.1$ \\
\hline
\end{tabular}

TABLE 1 shows an estimate of the heat leak into the MICE absorber with sixteen layers of MLI on the absorber body, pipes, a copper strap from the second stage cold head to the absorber and a liquid surge tank (with condenser). The heat leak estimate in TABLE 1 assumes that there is no intermediate temperature shield between the focusing-magnet warm bore and the absorber. The focusing magnet warm bore is assumed to be at $293 \mathrm{~K}$.

The absorber cold mass support is assumed to carry a load of $3 \mathrm{~g}(500 \mathrm{~N})$ in the radial direction and $2000 \mathrm{~N}$ in the longitudinal direction. The forces that might occur during a quench from the absorber not being centered in the focus magnet are much lower than the design values used for the cold mass supports. It is also assumed that there is no intermediate temperature intercept on the cold mass supports for the absorber.

The heat load into the absorber shown in TABLE 1 is low enough to permit liquid helium operation of the MICE absorber. The absorber cold mass support and insulation system design is continuing. It is hoped that the heat leak into the absorber can be reduced by almost a factor of two by having a shield around the absorber and an intermediate temperature support on the longitudinal cold mass support. The heat load into the first stage of the cooler is quite low. There appears to be plenty of excess first-stage cooling capacity to permit the pre-cooling of the hydrogen gas during absorber filling. Some of the extra cooling capacity can be used to cool an absorber shield.

\section{CONNECTION OF THE COOLER TO THE ABSORBER}

The most common method for connecting a small cooler to whatever it is cooling is to use a copper strap between the cooler and the absorber. The $\square \mathrm{T}$ along a copper strap is inversely proportional to the strap thermal conductance. The strap thermal conductance is proportional to the strap average thermal conductivity times its cross-sectional area and is inversely proportional to the length of the strap. In other words a longer copper strap will have a larger $\square \mathrm{T}$ for a given heat load from the absorber to the cooler. At $20 \mathrm{~K}$ the temperature drop between the absorber and the cold head is not much of a problem because there is excess temperature margin for liquid hydrogen operation and the thermal conductivity of pure copper is quite large in the temperature range from 10 to $20 \mathrm{~K}$.

At $4 \mathrm{~K}$, the conductance of the copper strap causes a large $\square \mathrm{T}$ between the absorber and the second stage cold head (relative to the temperature margin of the cooler), because OFHCcopper thermal conductivity is a factor of four lower at $4 \mathrm{~K}$ than it is at $20 \mathrm{~K}$. At $4 \mathrm{~K}$, it is not acceptable to use a copper strap to connect the second stage of the cooler to the load, except for the purpose of cooling down the absorber body to 5 or $6 \mathrm{~K}$.

An alternative method of connecting the cooler to the absorber is to use a gravity heat pipe. Liquid hydrogen (or helium) is boiled in the absorber so the gas goes from the top the absorber to a condenser that is connected to the second-stage cold head. The gas condenses on the condenser. The liquid from the condenser is fed to the bottom of the absorber. The fluid density difference circulates the liquid. A heat pipe can have a very low $\square \mathrm{T}(<0.1 \mathrm{~K})$ between the absorber and the cooler cold head. FIGURE 3 shows the connection between the cooler and the liquid absorber using a heat pipe. A copper strap shown is for absorber cooldown. FIGURE 4 shows the location of liquid-level gauges in the liquid buffer volume. 


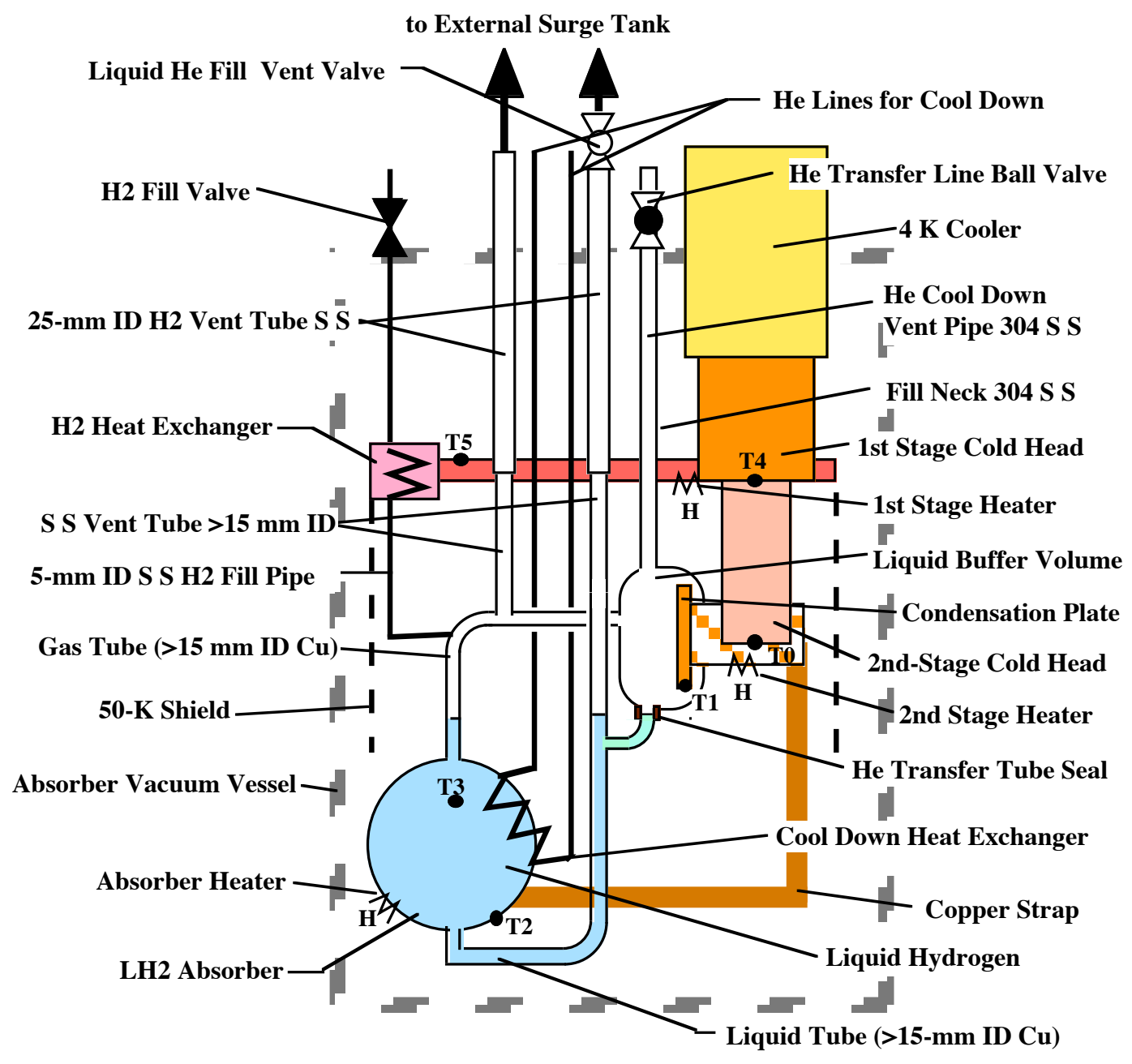

FIGURE 3. A schematic representation of the of the absorber within the absorber vacuum vessel. Included are the absorber, the condenser liquid surge volume, the hydrogen vent lines, and the absorber cool down lines, the two-stage 4-K cooler, and the absorber vacuum vessel. Temperature sensors and control heaters are shown.

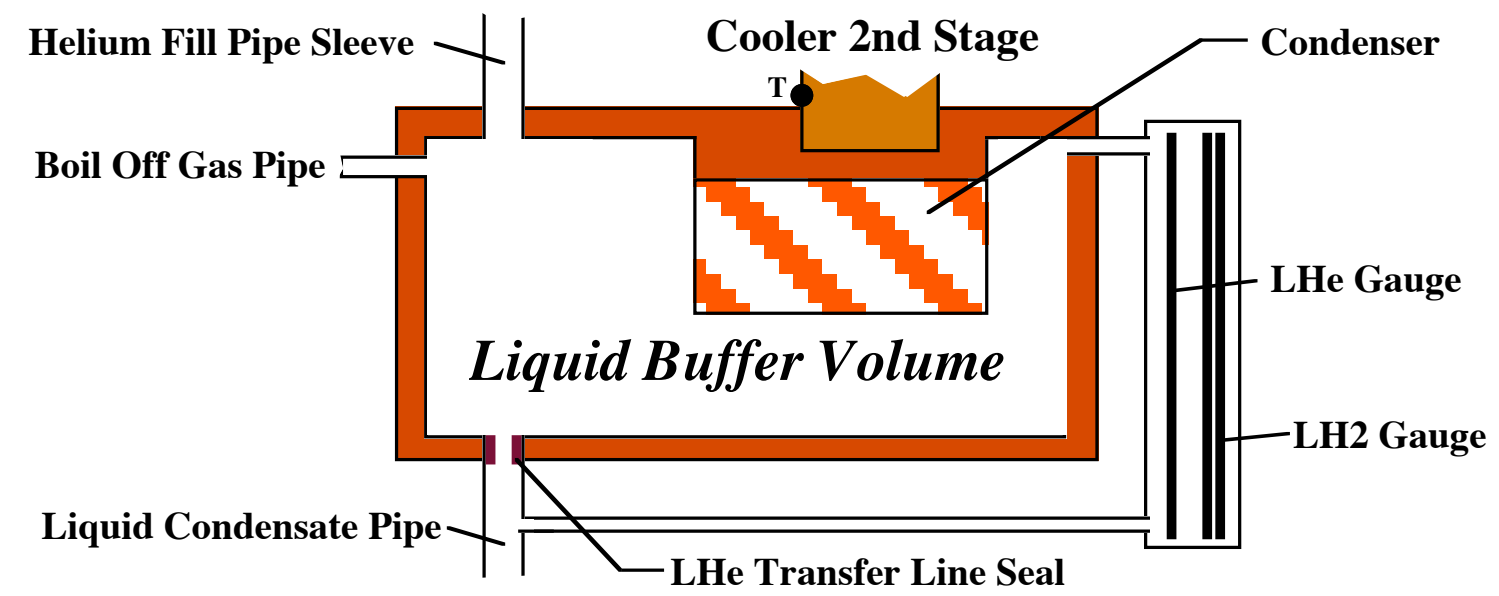

FIGURE 4. A schematic view of the liquid-hydrogen (helium) buffer volume. The buffer volume includes an extended surface condenser. The liquid leaves the buffer volume through the liquid pipe. The boil off gas enters through the gas pipe. The buffer volume includes the liquid hydrogen and liquid helium level sensors that can be used to control the liquid level within the absorber. 
TABLE 2. The operating parameters for the MICE absorber system for liquid hydrogen and liquid helium.

\begin{tabular}{lcc}
\multicolumn{1}{r}{ Operating Parameter } & LHe & $\mathrm{LH}_{2}$ \\
\hline \hline Maximum T at 0.17 MPa (K) & 4.82 & 22.1 \\
Triple point temperature (K) & 2.17 & 13.8 \\
Fill temperature (K) & 4.4 & 20.8 \\
Fill pressure (MPa) & 0.12 & 0.12 \\
Maximum absorber temp. (K) & 4.6 & 21.0 \\
Minimum absorber temp. (K) & 3.8 & 15.0 \\
Liquid volume change (liters) & 2.65 & 1.79 \\
\hline
\end{tabular}

TABLE 2 shows the absorber operating parameters for the liquid absorber filled with liquid helium or liquid hydrogen. The lowest possible operating temperature for a hydrogen absorber must be above the triple-point temperature of para-hydrogen. From TABLE 2, one can see that the liquid helium volume change determines the surge volume of the condensersurge tank. The effective volume of the surge tank can be about 2 liters. The range of hydrogen temperatures shown in TABLE 2 can be accommodated by a 2-liter tank. The temperature range for helium operation must be reduced (from $4.0 \mathrm{~K}$ to $4.6 \mathrm{~K}$ ). The condenser in the surge tank must not be covered by either liquid during normal operation.

The temperature of the second-stage cold head is used to control the liquid level in the absorber system. The liquid-level is measured by the sensors within the surge volume. The absorber is filled with hydrogen at $20.8 \mathrm{~K}$ to a set level within the surge volume. As the cooler cools the liquid hydrogen it contracts, causing the level to drop. When the liquid level reaches the bottom of the surge volume, a heater at the absorber or at the second stage cold head is turned on, which keeps the hydrogen level from falling further. The same principle is used to control the absorber system liquid level when helium is in the absorber.

\section{ABSORBER COOL-DOWN AND THE FILL WITH HYDROGEN OR HELIUM}

There are three methods of cooling down the MICE absorber. The first method is to cool the absorber by circulating liquid nitrogen and helium in the absorber heat exchanger. The second method is to cool the absorber down using the cooler. An extension of the second method is to liquefy hydrogen from gas entering the absorber from the hydrogen system. The third method is to inject liquid hydrogen directly into the absorber using the liquid fill system proposed for liquid helium.

Using liquid nitrogen to cool the absorber works well for getting the absorber temperature down to $80 \mathrm{~K}$. The cool down from $80 \mathrm{~K}$ to $20 \mathrm{~K}$ is a bit more complicated, particularly if the distance from the helium supply dewar to the absorber is long. Circulation of liquid helium from a pressurized storage-dewar is unstable, but cooling down with liquid helium from a dewar is not impossible [9]. There are two issues with using liquid helium to cool down the absorber and liquefy the hydrogen in the absorber. The first is that liquid temperature for helium at $0.1 \mathrm{MPa}(4.2 \mathrm{~K})$ is less than the triple-point temperature for hydrogen $(13.8 \mathrm{~K})$. This means that hydrogen will be frozen in the absorber if one is not careful. The second issue is the pre-cooling of the hydrogen gas before it is liquefied in the absorber. Pre-cooling the hydrogen removes up to 90 percent of the sensible heat from the gas. By pre-cooling the hydrogen, less helium is needed to liquefy the hydrogen. This is why it is recommended that hydrogen entering the absorber vacuum vessel be pre-cooled using the first stage of the cooler. Once the absorber has been cooled down and filled, the heat exchanger must be evacuated in order to reduce the heat load into the absorber.

The mass of the absorber body and windows is $\sim 19 \mathrm{~kg}$. One can cool the absorber from $293 \mathrm{~K}$ to $20 \mathrm{~K}$ using the cooler alone, provided the cooler is connected directly to the absorber using an OFHC copper strap. If the copper strap is $0.65 \mathrm{~m}$ long with a crosssectional area of $0.0005 \mathrm{~m}^{2}$, the cool down time to $20 \mathrm{~K}$ will be about 19 hours [4]. The cooling down of an absorber through a copper strap is like pumping down a vacuum system through a long pipe. The conductance of the copper strap is the key to decreasing the cool 
down time for the absorber body. If the strap cross-sectional area is reduced to $0.0002 \mathrm{~mm}^{2}$, the cool down time will increase about a factor of two. Liquefaction of hydrogen into the absorber can be done using the cooler. Most of the liquefaction will occur on the condenser within the liquid surge volume. When the cooler is used to liquefy the hydrogen entering the absorber, the hydrogen entering the absorber must be pre-cooled on the first stage of the cooler. A laminar flow heat exchanger with an area of $0.032 \mathrm{~m}^{2}$ attached to the cooler first stage will reduce the absorber liquid-hydrogen fill time from 106 hours to 24 hours. The calculated fill times includes the $3 \mathrm{~W}$ of heating due to the conversion of ortho hydrogen to para hydrogen. It will take about one week for all of the ortho hydrogen to be converted.

Since, the available excess refrigeration at $4.6 \mathrm{~K}$ is low, the liquefaction of helium into the absorber is not viable. Liquid helium from an external tank must be used to fill the absorber system. The same liquid injection system could be used to fill the absorber with hydrogen, but for safety reasons, this is not the preferred way of cooling down and filling the absorber with hydrogen. FIGURE 5 shows how a liquid absorber can be cooled down and filled with liquid helium from an external storage dewar. In FIGURE 5, the helium transfer line is shown as passing through a $12.7 \mathrm{~mm}$ ball valve. The tip of the transfer line mates with a seal in the bottom of the liquid buffer volume. Liquid helium is forced directly into the absorber from the bottom. After the helium transfer is finished, the transfer line is removed and the ball valve is closed. Once the absorber has been filled with helium, one must wait some time before the cooler can keep the helium liquid level at the right position.

The only hydrogen safety implication of using a small cooler is the possibility of freezing hydrogen on the condenser (in the event of a heater failure). The vent pipes are located well away from the condenser and dual heaters are used to control the liquid hydrogen level (See FIGURE 3). Helium operation does have safety implications not found with a pure liquid hydrogen absorber. Helium has a lower heat of vaporization than hydrogen $\left(20 \mathrm{~J} \mathrm{~g}^{-1}\right.$ versus $440 \mathrm{~J} \mathrm{~g}^{-1}$ ). The heat transfer coefficient for film boiling is also higher for helium. As a result, the mass flow of helium in the event of an accident is higher. The hydrogen vent system has to be designed to handle a much larger helium mass flow during a fault.

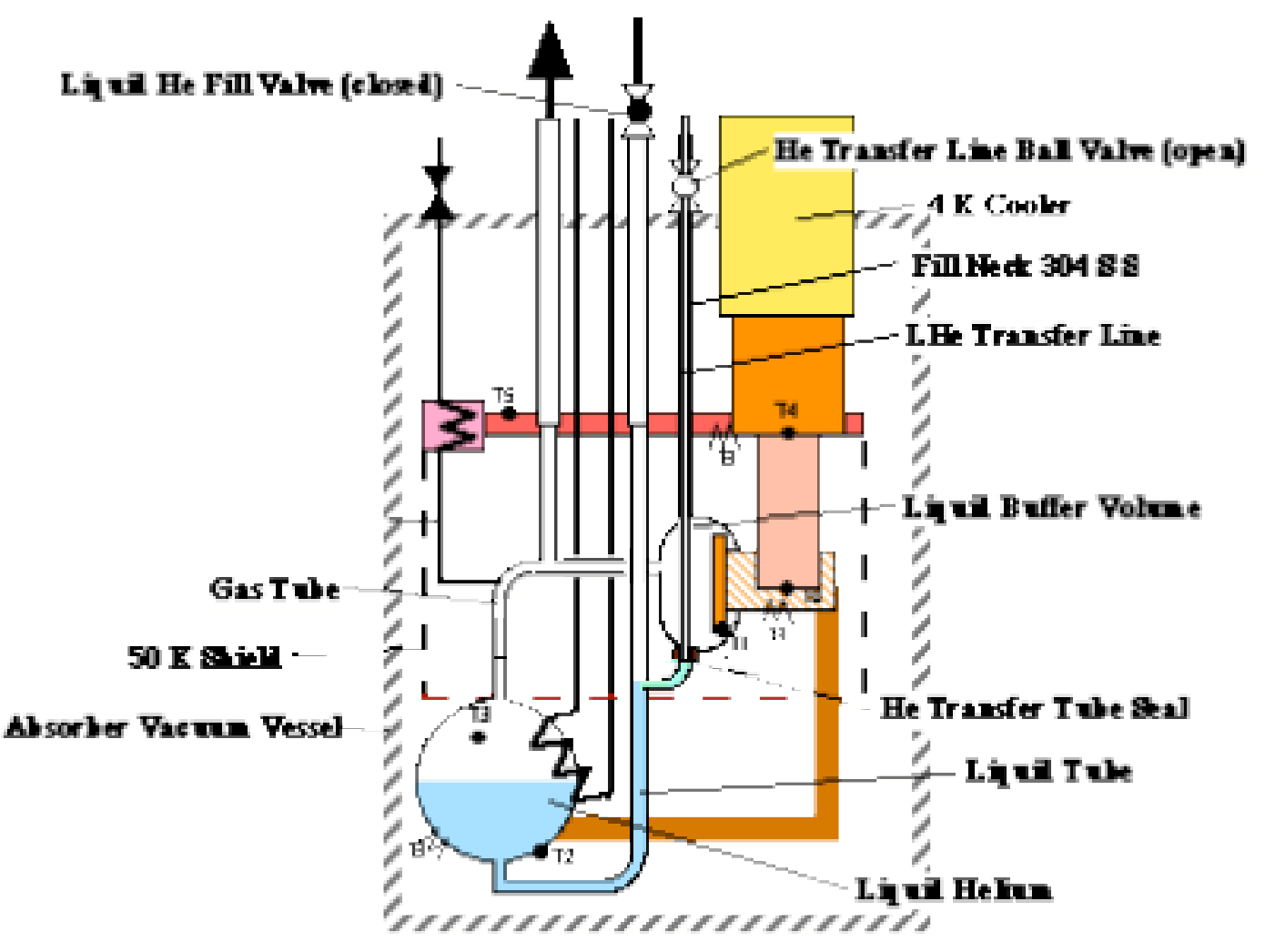

FIGURE 5. The process of cooling down and filling the MICE liquid absorber with liquid helium (or hydrogen) directly from a storage dewar. A valve on the vent line on the liquid side of the absorber is closed. Helium is vented from the gas side of the absorber to the external gas surge tank and the hydrogen vent. 


\section{CONCLUDING COMMENTS}

The cooling of the MICE liquid absorbers using a small cooler appears to be feasible for absorbers filled with liquid helium as well as liquid hydrogen. There is a refrigeration margin of about $16 \mathrm{~W}$ for either type of $4-\mathrm{K}$ cooler that might be considered for use on the MICE coolers. The refrigeration margin for liquid-helium operation is much lower (less than $1 \mathrm{~W}$ ) for both types of 4-K coolers. It appears that the absorber can be kept filled with helium from a storage dewar for short MICE runs with helium in the absorbers, even if the $4 \mathrm{~K}$ cooler doesn't quite hold the load. Further engineering is needed to reduce the heat load to the helium cooled absorber system. One approach to reducing the heat leak is to use the first stage of the cooler to cool a radiation shield on the inner bore of the focusing magnet.

For liquid-hydrogen operation, the absorber can be kept cold by using an OFHC copper strap between the absorber and the second stage of the cooler. The temperature drop along the strap is not a factor because the absorber can operate at temperatures from 15 to $21 \mathrm{~K}$. A copper strap cannot be used to carry the heat from the absorber to the cooler cold head when the absorber is filled with liquid helium. The $\square \mathrm{T}$ between the absorber and the cold head is too high. The use of a gravity feed heat pipe permits one to reduce the $\square \mathrm{T}$ between the absorber and the cold head to about $0.1 \mathrm{~K}$ with helium in the absorber. The same heat pipe system can be used when hydrogen is in the absorber with a lower $\square \mathrm{T}$.

The absorber can be cooled down passing liquid nitrogen and helium through the heat exchanger in the absorber body. The absorber can be cooled to liquid hydrogen temperature in about 15 hours using the cooler alone as long as the conductance of the copper strap carrying the heat is high enough. The cooler can liquefy hydrogen and fill the absorber, provided the hydrogen being liquefied is pre-cooled using the first stage of the cooler. For liquid-helium operation, liquid-helium must be transferred directly into the absorber from a storage dewar. The absorber can be filled with hydrogen from a storage dewar as well.

The safety issues created by using a small cooler to cool the MICE absorber system can be taken care of by proper positioning of the vent line, by using dual heaters to control the absorber liquid level, and by the proper sizing of the relief devices on the absorber and its vacuum vessel.

\section{ACKNOWLEDGEMENTS}

This work was supported by the Oxford University Physics Department and the Particle Physics and Astronomy Research Council of the United Kingdom. A portion of this work was done with the support of the Office of High Energy Physics, United States Department of Energy under DOE contract DE-AC02-05CH11231.

\section{REFERENCES}

1. "A Proposal to the Rutherford Appleton Laboratory, an International Muon Ionization Cooling Experiment" a MICE Collaboration proposal, http://hep04.phys.itt.edu/cooldemo (15 Dec 2002).

2. Green, M. A., “The Cryogenic Refrigeration System for MICE," LBNL-51751, (Nov. 2002).

3. Green, M. A., "Cooling the MICE Magnets using Small Cryogenic Coolers," Oxford University Physics Department Note 10, MICE Note 109, http://hep04.phys.itt.edu/cooldemo, (Sept. 2004).

4. Green, M. A., "Cooling the MICE Liquid Hydrogen Absorbers using Small Cryogenic Coolers," Oxford University Physics Department Note 9, MICE Note 108, http://hep04.phys.itt.edu/cooldemo, (2004).

5. Baynham, D. E., Bradshaw, T., Cummings, M. A., et al., "A Liquid Absorber for MICE," Advances in Cryogenic Engineering 51, AIP Press, Melville NY (This publication).

6. Data sheets for the RDK-415D and the RDK-408S GM coolers from Sumitomo Industries.

7. Data Sheets for the PT-410 and the PT810 pulse tube coolers from Cryomech Incorporated.

8. Green, M. A., "Integration of Liquid Cryogen Cooling and Cryo-coolers with Superconducting Electronic Systems," Superconducting Science and Technology, ISEC-2003 Special Issue, (2003).

9. Ishimoto, S., a report to the MICE collaboration of the MUCOOL absorber liquid hydrogen tests at Fermi National Laboratory in 2004, http://hep04.phys.itt.edu/cooldemo, 


\section{DISCLAIMER}

This document was prepared as an account of work sponsored by the United States Government.

While this document is believed to contain correct information, neither the United States Government nor any agency thereof, nor The Regents of the University of California, nor any of their employees, makes any warranty, express or implied, or assumes any legal responsibility for the accuracy, completeness, or usefulness of any information, apparatus, product, or process disclosed, or represents that its use would not infringe privately owned rights. Reference herein to any specific commercial product, process, or service by its trade name, trademark, manufacturer, or otherwise, does not necessarily constitute or imply its endorsement, recommendation, or favoring by the United States Government or any agency thereof, or The Regents of the University of California. The views and opinions of authors expressed herein do not necessarily state or reflect those of the United States Government or any agency thereof, or The Regents of the University of California. 